\title{
Efficacy and follow-up of transcatheter aortic valve implantation in a patient with radiation-induced aortic stenosis accompanied by extensive calcification and restrictive pulmonary dysfunction as radiotherapy complications
}

\author{
Gultekin NN*, Yildi A, Kocas C, Burcu Topcu and Ökcun EB \\ Istanbul University Cerrahpasa, Cardiology Institute, Department of Cardiology, Istanbul, Turkey
}

\begin{abstract}
We present a case of Hodgkin's lymphoma with a radiation-induced severe calcific aortic stenosis accompanied by diffuse calcifications as a complication of radiotherapy with severe exertional dyspnea was in last 6 months and 2 syncope attack in last month, also a severe restrictive pulmonary disease secondary to mediastinal radiotherapy. The Society of Thoracic Surgery (STS) risk score was $5.2 \%$ with a severe restrictive pulmonary disease. The heart valve team in our unit decided to perform Transcatheter Aortic Valve Implantation (TAVI) due to patient-specific risk factors for surgical aortic valve replacement.Core Valve Evolut ${ }^{\text {TM }} \mathrm{R}$ $26 \mathrm{~mm}$ valve selected according to diameters. EvolutR ${ }^{\text {TM }} 26 \mathrm{~mm}$ valve positioned and the procedure was finished without aortic regurgitation. Patient follow-up was uneventful for 16 months.
\end{abstract}

\section{Introduction}

Patients with prior mediastinal radiotherapy (XRT) for thoracic malignancies frequently develop radiation-associated cardiac disease. Valvular heart disease occurs in $\approx 81 \%$ of patients with radiation-associated cardiac disease, and a significant proportion present with severe symptomatic aortic stenosis (AS) requiring an intervention. While aortic or mitral valvular regurgitation is the more commonly seen dysfunction, aortic stenosis is typically the main reason motivating surgical options .Transcatheter Aortic Valve Implantation (TAVI) appears to be a highly promising procedure with high feasibility, specific early complications, positive postprocedural haemodynamic outcomes assessed by echocardiography, and lower mid-term mortality when compared to SAVR [1-4].

\section{Case report}

We present a case of Hodgkin's lymphoma with a radiationinduced severe calcific aortic stenosis accompanied by diffuse calcifications as a complication of radiotherapy. Hodgkin's lymphoma developed in this patient and chemotherapy and radiotherapy were applied when he was three year old and also, performed splenectomy when he was eight years. Thyroidectomy was performed at 18 years old and developed hypoparathyroidism. Now, he was 49-year old and admitted to our unit with severe exertional dyspnea for six months of ongoing and two syncope attack in last month. Also, there was severe restrictive pulmonary disease (secondary to mediastinal radiotherapy) in this patient. He was started on treatment; furosemide $40 \mathrm{mg}$ every-other-day, ramipril $5 \mathrm{mg} 1 \mathrm{x} 1$, metoprolol $50 \mathrm{mg} 1 \mathrm{x} 1,1,25$ dihydroxycholecalciferol (calcitriol) $0.5 \quad 1 \times 1$,calcimax-D3 $2 \times 1$ daily. Physical examination: Consciousness was open and cooperated.
Blood pressure: 120/75 mmHg. Pulse 66/min. There was an ejection murmur spreading to all foci from the mesocardiac area. Laboratory findings: Biochemical tests were normal. The heart cavities were of normal width. There was global ventricular hypertrophy. Global hypokinesia was present (Ejection Fraction: 50\%). Aortic valve thick, the opening was restricted. Aortic root width was 3.1, Inter Ventricular Septum Thickness: 1.3, Left Ventricular Posterior Wall Thickness: $1.2 \mathrm{~cm}$, Left Ventricular Diastolic Diameter: $4.5 \mathrm{~cm}$. Aortic valve area $1 \mathrm{~cm}^{2}$, mean 40 , peak $70 \mathrm{mmHg}$ gradient and medium aortic insufficiency flow were obtained with the Doppler examination. The structure and movement of the other valves were normal. Mild tricuspid insufficiency flow was obtained with the doppler examination. E/A ratio was increased in favour of A, IVRT, DT prolonged (Figures $1 \mathrm{~A}$ and $1 \mathrm{~B}$ ). In electrocardiography (ECG) was rhythm sinus (80/minute), LVH findings were present. In pulmonary function tests FEV: $30 \%$ showed an advanced restrictive pattern. Multislice CT coronary angiography revealed normal coronary arteries, pericardial and aortic calcifications. It was noteworthy around two $\mathrm{cm}$ diameter craniocaudal calcifications on aortic valve and ascending aorta, and was extending from anterior to posterior of the pericardium,

*Correspondence to: Gultekin NN, Istanbul University Cerrahpasa, Cardiology Institute, Department of Cardiology, Istanbul, Turkey, E-mail: nngultekin@yahoo.com

Key words: transcatheter aortic valve implantation, radiotherapy, aortic calcifications, multislice computed tomography angiography

Received: March 19, 2019; Accepted: March 26, 2019; Published: March 29, 2019 
Gultekin NN (2019) Efficacy and follow-up of transcatheter aortic valve implantation in a patient with radiation-induced aortic stenosis accompanied by extensive calcification and restrictive pulmonary dysfunction as radiotherapy complications

which is believed to be compatible with radiotherapy collimation windows. Multislice CT performed to determine aortic diameters (Figures 1C-1E). Transcatheter Aortic Valve Implantation-Surgical Aortic Valve Replacement (TAVI-SAVR): The Society of Thoracic Surgery (STS) score was $5.2 \%$, severe restrictive pulmonary disease, severe calcific aortic stenosis accompanied by diffuse calcifications as a complication of radiotherapy. The heart valve team in our unit decided to perform TAVI due to patient-specific risk factors for SAVR.

TAVI procedure: Core Valve Evolut ${ }^{\mathrm{mm}} \mathrm{R} 26 \mathrm{~mm}$ valve selected according to diameters. Evolut ${ }^{\mathrm{m}} \mathrm{R} 26 \mathrm{~mm}$ valve positioned and the procedure was finished without aortic regurgitation (Figures $2 \mathrm{~A}-2 \mathrm{~F}$ ) (Video 1). Follow-up 11 months: Patient follow-up was un-eventful for 16 months (Figures $2 \mathrm{G}$ and $2 \mathrm{H}$ ).

\section{Discussion}

This is the first report to focus on patients with TAVI, which is referred to our institution with radiation-induced aortic stenosis. We evaluated the patient during 11 months follow-up. As the main finding, we reduced the mortality rate in patients with radiation-induced aortic stenosis at 9 months. TAVI is a good choice for patients with mediastinal radiotherapy and porcelain aortic valve and patients with a restrictive pattern of advanced respiratory function. In patients with radiation-induced aortic valve stenosis and contraindications to conventional surgery conditions, TAVI appears to be a highly promising procedure with high feasibility, specific early complications, positive postprocedural haemodynamic outcomes assessed by
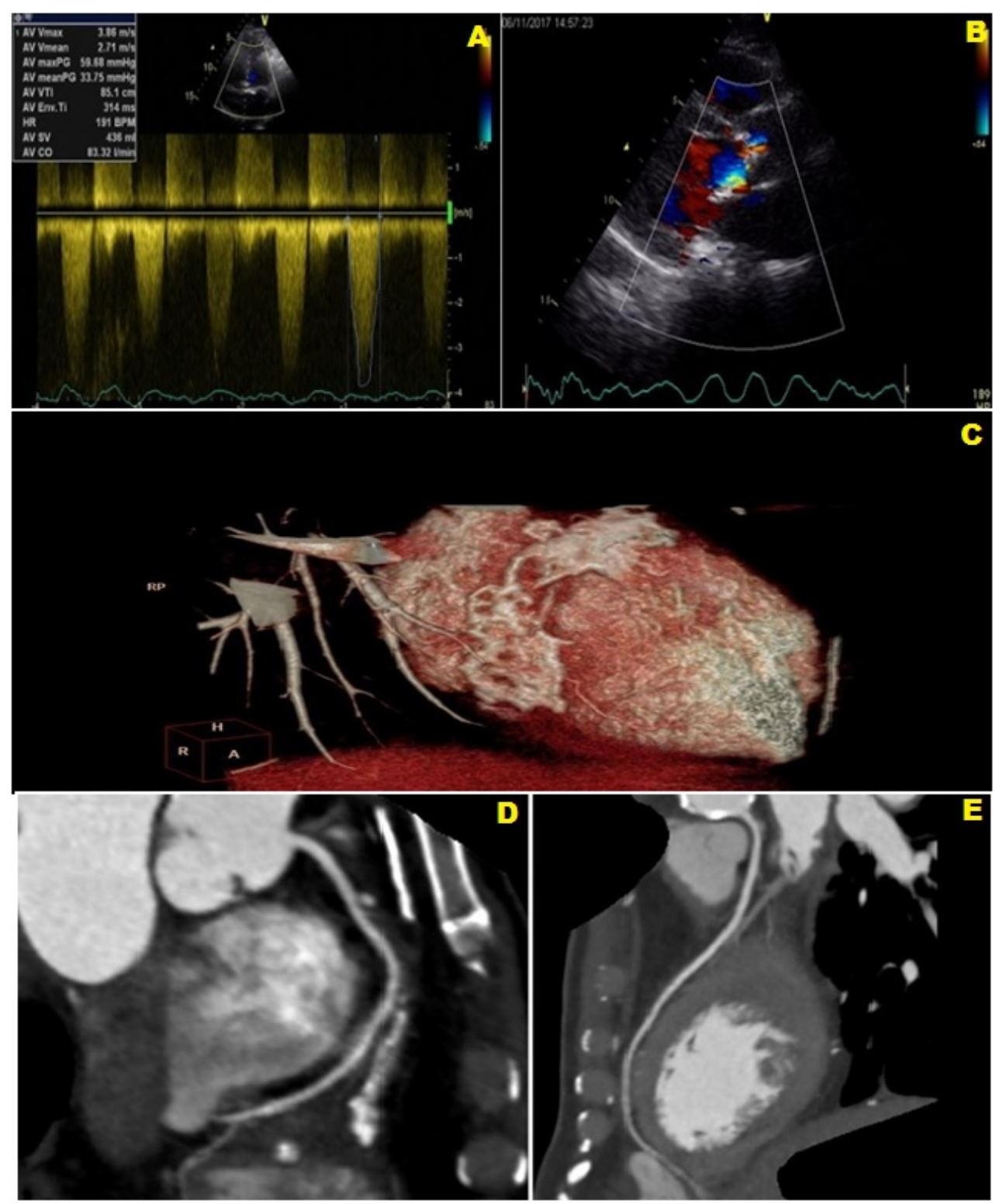

Figure 1 A, B. Baseline echocardiogram: Preserved LV function with significant LV hypertrophy Severe aortic stenosis. C. Multıslice CT: It was noteworthy around 2 cm diameter craniocaudal calcifications on aortic valve and ascending aorta, and is anteriorly and posteriorly of the pericardium, which is believed to be compatible with radiotherapy collimation windows. D,E. CT-Angiography: Multislice CT coronary angiography revealed normal coronary arteries 
Gultekin NN (2019) Efficacy and follow-up of transcatheter aortic valve implantation in a patient with radiation-induced aortic stenosis accompanied by extensive calcification and restrictive pulmonary dysfunction as radiotherapy complications
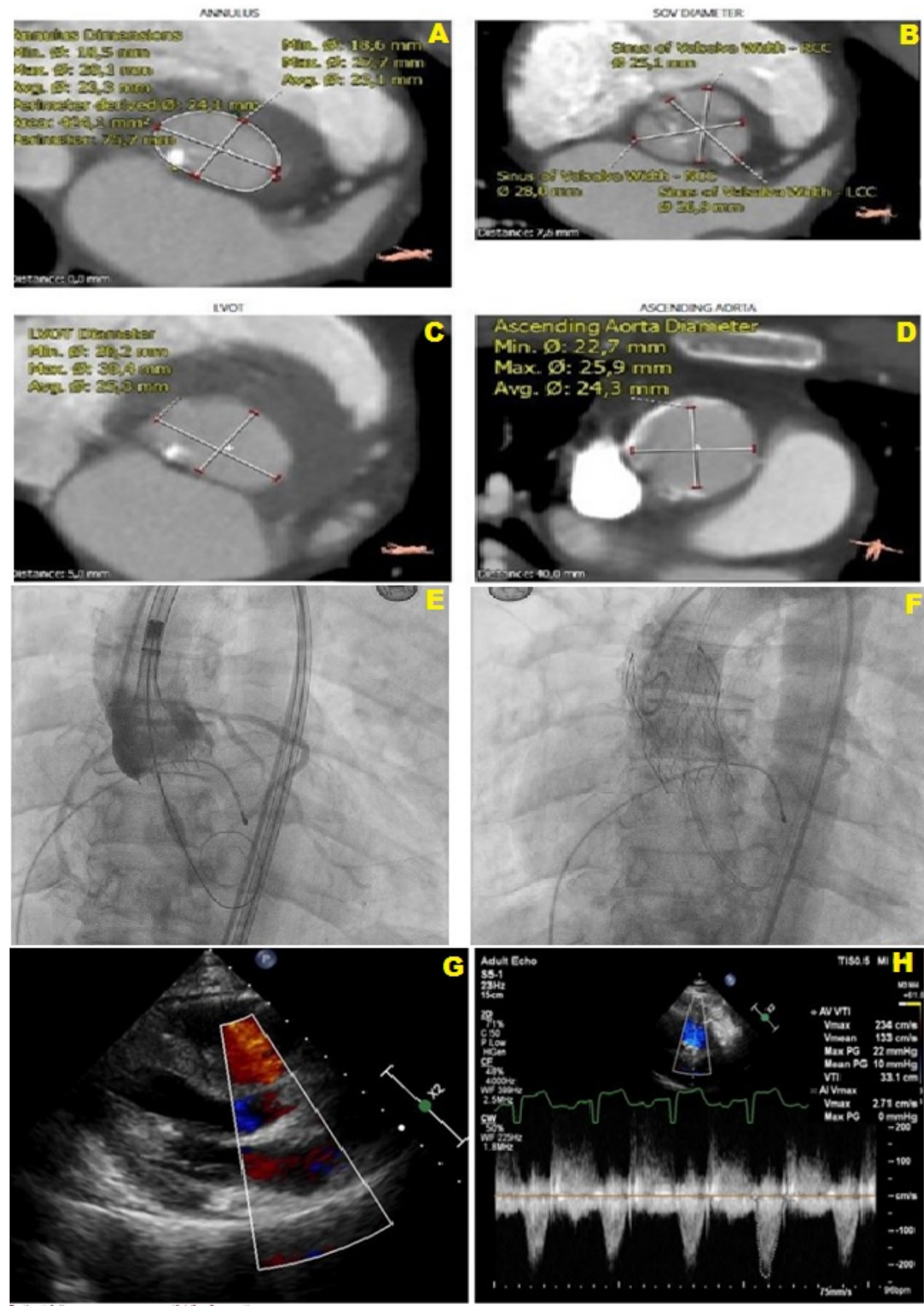

Figure 2.A-D. Multislice CT:Multislice CT performed to determine aortic diameter. E. TAVI procedure: Evolut -R 26 mm valve positioned...F. TAVI procedure:Procedure was finished without aortic regurgitation.G,H. Follow-up 11 month 
Gultekin NN (2019) Efficacy and follow-up of transcatheter aortic valve implantation in a patient with radiation-induced aortic stenosis accompanied by extensive calcification and restrictive pulmonary dysfunction as radiotherapy complications

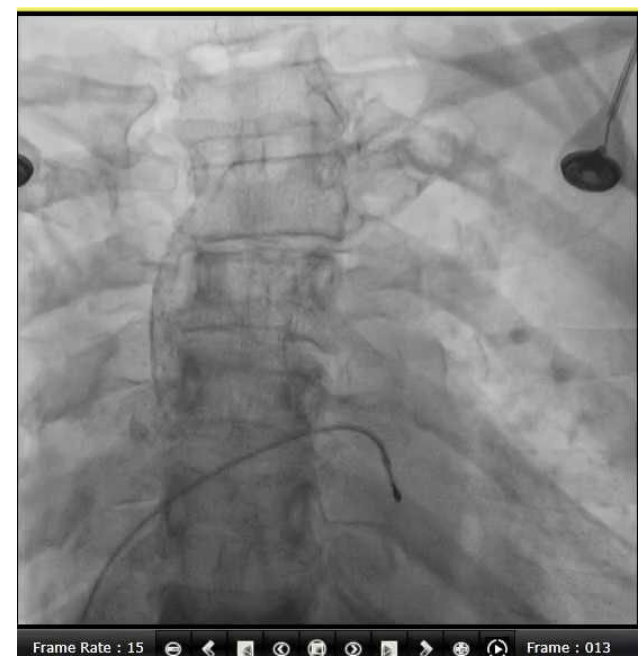

Video 1. Evolute R TM26 mm valve positioned

echocardiography, and lower mid-term mortality when compared to SAVR. In the studies, TAVI had high clinical efficacy, more than $85 \%$ of patients were classified as NYHA class 1 or 2 at 6 months, and no readmission to the valve was required [1-4].The effect of this intervention is better selection of subgroup cardio-oncology patients for TAVI even at younger ages despite the durability problem.

\section{Conclusion}

TAVI may be a good option for cardio-oncology patients with a radiation-induced severe calcific aortic stenosis accompanied by diffuse calcifications as a complication of previous mediastinal radiotherapy.

\section{References}

1. Donnellan E, Krishnaswamy A, Hutt-Centeno E, Johnston DR, Aguilera J, et al. (2018) Outcomes of Patients with Mediastinal Radiation-Associated Severe Aortic Stenosis Undergoing Transcatheter Aortic Valve Replacement. Circulation 138: 1752-54. [Crossref]

2. Hull MC, Morris CG, Pepine CJ, Mendenhall NP (2003) Valvular dysfunction and carotid, subclavian, and coronary artery disease in survivors of Hodgkin lymphoma treated with radiation therapy. JAMA 290: 2831-7. [Crossref]

3. Carlson RG, Mayfield WR, Normann S, Alexander JA (1991) Radiation-associated valvular disease. Chest 99: 538-545. [Crossref]

4. Dijos M, Reynaud A, Leroux L, Réant P, Cornolle C, et al. (2015) Efficacy and followup of transcatheter aortic valve implantation in patients with radiation-induced aortic stenosis. Open Heart 2: e000252. [Crossref]

Copyright: $@ 2019$ Gultekin NN. This is an open-access article distributed under the terms of the Creative Commons Attribution License, which permits unrestricted use, distribution, and reproduction in any medium, provided the original author and source are credited. 